\title{
Soluble maize fibre affects short-term calcium absorption in adolescent boys and girls: a randomised controlled trial using dual stable isotopic tracers
}

\author{
Corrie M. Whisner ${ }^{1}$, Berdine R. Martin ${ }^{1}$, Cindy H. Nakatsu ${ }^{2}$, George P. McCabe ${ }^{3}$, Linda D. McCabe ${ }^{3}$, \\ Munro Peacock ${ }^{4}$ and Connie M. Weaver ${ }^{1 *}$ \\ ${ }^{1}$ Department of Nutrition Science, Purdue University, 700 West State Street, West Lafayette, IN 47907, USA \\ ${ }^{2}$ Department of Agronomy, Purdue University, 700 West State Street, West Lafayette, IN 47907, USA \\ ${ }^{3}$ Department of Statistics, Purdue University, 700 West State Street, West Lafayette, IN 47907, USA \\ ${ }^{4}$ Department of Medicine, Indiana University School of Medicine, Indianapolis, IN, USA
}

(Submitted 22 August 2013 - Final revision received 28 March 2014 - Accepted 31 March 2014-First published online 22 May 2014)

\begin{abstract}
Soluble maize fibre (SCF) has been found to significantly improve bone mineral density and strength in growing rats compared with several other novel prebiotic fibres. The objective of the present study was to investigate the effect of SCF on Ca absorption and retention in pubertal children by studying the potential absorption mechanisms of the intestinal microbiota. A total of twenty-four adolescent boys and girls (12-15 years) participated in two 3-week metabolic balance studies testing $0 \mathrm{~g} / \mathrm{d} \mathrm{SCF}$ (control (CON) treatment) and $12 \mathrm{~g} / \mathrm{d}$ SCF (SCF treatment) in a random order by inclusion in a low-Ca diet $(600 \mathrm{mg} / \mathrm{d})$. Fractional Ca absorption was measured at the end of the two intervention periods using a dual-stable isotope method. Diet composites and faecal and urine samples were collected daily and analysed for Ca content. Ca retention was calculated as dietary Ca intake minus Ca excretion in faeces and urine over the last 2 weeks. Microbial community composition in the faecal samples collected at the beginning and end of each session was determined by 454 pyrosequencing of the PCR-amplified $16 S$ ribosomal RNA gene. Fractional Ca absorption was $12 \%$ higher $(41 \mathrm{mg} / \mathrm{d})$ after the SCF treatment compared with that after the CON treatment (0.664 (SD 0.129) and 0.595 (SD 0.142), respectively; $P=0.02$ ), but Ca retention was unaffected. The average proportion of bacteria of the phylum Bacteroidetes was significantly greater in the participants after the SCF treatment than after the CON treatment. These results suggest that moderate daily intake of SCF, a well-tolerated prebiotic fibre, increases short-term Ca absorption in adolescents consuming less than the recommended amounts of $\mathrm{Ca}$.
\end{abstract}

Key words: Prebiotics: Calcium absorption: Microbiota: Soluble maize fibre

Adolescence is an important stage of life for bone health that provides a unique opportunity to maximise mineral retention and prevent the risk of osteoporosis-related fractures later in life. Because $\mathrm{Ca}$ is becoming increasingly deficient in the diet due to a decrease in milk consumption ${ }^{(1,2)}$, there is strong interest in functional food ingredients for enhancing Ca utilisation. Non-digestible oligosaccharides, including fructo-oligosaccharides $^{(3,4)}$ and galacto-oligosaccharides ${ }^{(5)}$, have been reported to be associated with increased $\mathrm{Ca}$ absorption. In animal studies, these carbohydrate compounds have been found to be associated with increases in bone mineral density $(\mathrm{BMD})^{(6-8)}$ as well as improved bone geometry and strength ${ }^{(7,9,10)}$. The increase in Ca absorption and subsequent improvements in bone health are possibly mediated by microbial fermentation. Bongers \& Van den $\mathrm{Heuvel}^{(11)}$ suggested that SCFA produced by microbial fermentation can produce an acidic intestinal environment ideal for increasing the solubility and transcellular absorption of Ca.

We had compared eight novel fibres for their effects on $\mathrm{Ca}$ utilisation and bone properties in a growing rat model $^{(12)}$. Soluble maize fibre (SCF) was found to have effects similar to those of soluble fibre dextrin and confer greater bone health benefits when compared with resistant starch (RS), pullulan, polydextrose, inulin and Synergy $1{ }^{\circledR}$; ORAFTI. SCF increased bone mineral content by $7 \cdot 6 \%$, BMD by $2.9 \%$, femoral cortical area by $19 \cdot 6 \%$, cortical thickness by $22 \cdot 4 \%$ and peak breaking force by $8 \cdot 8 \%$. Compared with controls, SCF resulted in significant increases in faecal content weight and total SCFA, acetate and propionate production, which, in part, indicate the involvement of intestinal microbes.

Factors that enhance bone density and bone mineral content have the potential to contribute to a skeleton that

Abbreviations: BMD, bone mineral density; CON, control; IGFBP3, insulin-like growth factor-binding protein 3; NTX, cross-linked N-telopeptides of type I collagen; OTU, operational taxonomic units; rRNA, ribosomal RNA; RS, resistant starch; SCF, soluble maize fibre. 
will resist fractures later in life. Prebiotics may provide a novel means of influencing mineral bioavailability to improve peak bone mass. Therefore, the objective of the present study was to better understand the role of SCF in humans by examining its effects on Ca absorption and retention as well as changes in gut microbiota composition in adolescent boys and girls consuming habitually low daily $\mathrm{Ca}$ amounts.

\section{Subjects and methods \\ Subjects}

Healthy adolescent girls and boys were recruited from local schools, community centres and Indiana extension offices and by sending direct mails to the surrounding areas. A total of fifteen boys, aged 13-15 years, and nine girls, aged 1214 years, participated in the metabolic studies. Screening questionnaires were used to determine eligibility based on a brief medical history, maturational age, physical activity and habitual dietary intake assessed with a $6 \mathrm{~d}$ diet record. Adolescent girls and boys who were generally healthy and were willing to consume the foods provided as part of the controlled diets were eligible to participate in the study. Exclusion criteria included abnormal liver or kidney function, malabsorptive disorders, anaemia, smoking, history of using medications influencing Ca metabolism (steroids and thiazide diuretics), body weight outside the 5th-95th BMI percentile for age, regular consumption of illegal drugs, non-prescription drugs, or any kind of contraceptives, and pregnancy. The subjects were not permitted to take nutritional supplements while participating in these studies and were asked to discontinue use before coming to the camp. The present study was conducted according to the guidelines laid down in the Declaration of Helsinki, and all procedures involving human subjects were approved by the Institutional Review Board of Purdue University. Written informed consent was obtained from all subjects. The present study was registered at ClinicalTrials.gov ID: NCT01571440.

\section{Study design}

The present study, designed to provide a summer camp environment, consisted of two 3-week balance studies separated by a $7 \mathrm{~d}$ washout period. A double-blind, cross-over design was used in which the participants received two treatments in a randomised order: $12 \mathrm{~g}$ SCF (SCF treatment) or $0 \mathrm{~g}$ SCF (control (CON) treatment). The study staff used a simple technique for randomisation such that the treatment orders were drawn, for each participant, from a hat containing equal numbers of the two possible randomisation schemes. Studies were structured as summer camps where adolescent boys and girls went on field trips, participated in recreational activities and performed a variety of educational activities. The participants were housed in University Residence Halls at Purdue University during the camp duration.

\section{Diets}

Controlled diets were provided throughout both camp sessions and consisted of foods typically eaten by adolescent children such as spaghetti, hamburgers, sandwiches and potato chips. The participants were assigned to one of five energy levels (7322, 8786, 10042, 11297 and $12552 \mathrm{~kJ}$ ) based on the estimated energy requirements calculated using the Harris-Benedict equations ${ }^{(13)}$. The diets were designed to maintain body weight and to contain constant amounts of key nutrients. The controlled diets were provided as a $4 \mathrm{~d}$ cycle menu with three meals and two snacks daily. On average, the diets contained $14 \%$ protein, 33\% fat, 53\% carbohydrate, $5 \mu \mathrm{g}$ vitamin D, $1100 \mathrm{mg}$ P, $2300 \mathrm{mg} \mathrm{Na}$ and $600 \mathrm{mg}$ $\mathrm{Ca}$. The basal diet contained $15 \mathrm{~g}$ of fibre and the intervention product contributed an additional 0 or $12 \mathrm{~g} \mathrm{SCF}$. This yielded a total dietary fibre content of 15 and $27 \mathrm{~g}$ for the CON and SCF treatments, respectively. SCF was present in Welch's ${ }^{\circledR}$ fruit snacks (WELCH Foods, Inc.) and divided into two 0 or $6 \mathrm{~g}$ fibre doses provided at lunch and dinner. The SCF ingredient (PROMITOR ${ }^{\circledR}$ Soluble Corn Fiber 70 ) provided by Tate \& Lyle is a fermentable, non-digestible carbohydrate and contains $70 \%$ (minimum) soluble dietary fibre.

\section{Anthropometric parameter and bone measurements}

Anthropometric parameters including weight, sitting height, bitrochanteric width, waist circumference and hip circumference were measured during the first session of the camp. Standing height was measured at the beginning of the first session using a wall-mounted stadiometer, and weight was monitored in the morning on each day with an electronic digital scale to ensure that it remained stable throughout the sessions. Bone mineral content and BMD were measured by dualenergy X-ray absorptiometry (GE Lunar) during one balance period to determine the bone status of the participants. Bone measurements of the whole body, spine, forearm and both hips were taken.

\section{Measurement of hormones and biochemical markers of bone metabolism}

A fasting, baseline blood sample was collected on the 1st day of the camp for the determination of general blood chemistry to verify the clinical profile and health of the participants. A second fasting sample was collected at the end of the camp to measure the biochemical markers of bone dynamics and hormones related to $\mathrm{Ca}$ and vitamin $\mathrm{D}$ metabolism. The concentrations of serum total $\mathrm{Ca}$, phosphate, total alkaline phosphatase and creatinine and urinary creatinine and phosphate were measured by routine chemistry. The concentration of serum parathyroid hormone 1-84 was determined using a two-site immunoassay (CV $\pm 7 \cdot 1 \%$ ) (Nichols Institute Diagnostics). The concentrations of insulin-like growth factor 1 and insulin-like growth factor-binding protein 3 (IGFBP3) were measured by ELISA (Diagnostics Systems Laboratories, Inc.). The concentration of leptin was determined by RIA (EMD Millipore) and that of sclerostin by ELISA (TECOmedical AG). The concentrations of urinary cross-linked N-telopeptides of type I collagen (NTX) were measured by ELISA (Ostex International). The concentration of serum 
25-hydroxyvitamin D was determined by LC-MS/MS analysis (Agilent Rapid Res 1200 LC system, Novilytic LLC).

\section{Sample collection and analysis}

All urine and faecal samples were collected from day 1 to day 21 of each balance period and pooled as $24 \mathrm{~h}$ collections. $\mathrm{Ca}$ content in the diets and faecal and urine samples was measured using inductively coupled plasma optical emission spectrometry (Optima 4300 DV, Perkin Elmer Instrument) as described previously ${ }^{(14)}$. All faecal samples were frozen and processed later for the determination of $\mathrm{Ca}$ content. Urine samples were refrigerated and also analysed later for the determination of total $\mathrm{Ca}$ content. The procedure followed for faecal sampling for the microbiological analysis is described below.

\section{Evaluation of compliance}

The participants were supervised during activity, meal and sample collection periods by trained counsellors for $24 \mathrm{~h}$ each day. Unconsumed food from meals was collected and its amount recorded. Urine sample collection compliance was evaluated by measuring creatinine excreted in the urine by an enzymatic colorimetric assay (COBAS Integra, Roche Diagnostics). Faecal sample collection compliance was assessed by polyethylene glycol recovery in the faeces. Each participant was given $3 \mathrm{~g}$ of polyethylene glycol (E3350; Dow Chemical Company) divided into $1 \mathrm{~g}$ doses at breakfast, lunch and dinner. The percentage of polyethylene glycol recovery was measured in $24 \mathrm{~h}$ faecal collections by a turbidimetric assay ${ }^{(15)}$ and was used as a basis to exclude participant data in case of poor compliance.

\section{Evaluation of gastrointestinal symptoms}

The presence of stomach noises, flatulence, bloating and abdominal pain among the participants was evaluated daily using a short questionnaire. The severity of gastrointestinal symptoms was assessed daily by self-reports using a scale of $1-10(0=$ none and $10=$ very severe $)$. The questionnaire was created during the second 3-week treatment, which did not allow for validation before use and resulted in a shorter assessment period (18 consecutive days).

\section{Fractional calcium absorption test}

During the last week of each session, following an overnight fast, the participants underwent a Ca absorption test carried out using methods described previously ${ }^{(16)}$. On the morning of the test, phlebotomists inserted catheters and drew $10 \mathrm{ml}$ baseline venous samples. Immediately after the blood draw, the participants consumed a breakfast consisting of an English muffin, scrambled eggs, butter and jam. The meal contained $150 \mathrm{mg}$ Ca from $2 \%$ milk plus $15 \mathrm{mg}{ }^{44} \mathrm{Ca}$ (96.5\% enrichment), a stable non-radioactive isotope. The oral isotope was administered as a liquid calcium chloride $\left({ }^{44} \mathrm{CaCl}_{2}\right)$ that was added to the milk and allowed to equilibrate overnight.
Following breakfast, the participants were not allowed to consume any food, but were allowed to drink ad libitum deionised water. A second stable isotope, ${ }^{43} \mathrm{Ca}(5 \mathrm{mg}, 60 \cdot 2 \%$ enrichment) also given as calcium chloride, was administered intravenously $1 \mathrm{~h}$ after the consumption of breakfast and the oral isotope. A final blood draw was performed $3 \mathrm{~h}$ after the administration of the intravenous dose, after which the catheters were removed and the participants were served lunch.

\section{Absorption and retention calculations}

Changes in fractional $\mathrm{Ca}$ absorption over $48 \mathrm{~h}$ following $\mathrm{Ca}$ isotope administration were measured using two $24 \mathrm{~h}$ urine pools with a modified version of the published methodologies $^{(16)}$. Urine samples collected in $24 \mathrm{~h}$ pools for $2 \mathrm{~d}$ (0-24 and 24-48h) after the absorption test were analysed for ${ }^{44} \mathrm{Ca}$ and ${ }^{43} \mathrm{Ca}$ enrichment by high-resolution inductively coupled plasma MS (Finnegan Element2, Thermo Scientific). To more specifically measure increases in absorption due to bacterial fermentation in the colon, two separate $24 \mathrm{~h}$ pooled analyses were used instead of using the more typical $48 \mathrm{~h}$ cumulative urine analysis. This method is based on previous results indicating that a urine collection period of at least $36 \mathrm{~h}$ is required to detect an effect of prebiotics ${ }^{(17)}$. Previously, our laboratory group had found that separating urine samples into $12 \mathrm{~h}$ pools was helpful in identifying the 24-48 h time frame as being important for measuring lower gut absorption $^{(16)}$. Fractional Ca absorption (equation 1) was calculated as an enrichment ratio of the administered isotopes $\left({ }^{44} \mathrm{Ca}\right.$ and $\left.{ }^{43} \mathrm{Ca}\right)$ to another naturally occurring isotope $\left({ }^{42} \mathrm{Ca}\right)$ in the $0-24$ and $24-48 \mathrm{~h}$ urine collections. The $\Delta$ excess value was calculated as the presence of ${ }^{44} \mathrm{Ca}$ and ${ }^{43} \mathrm{Ca}$ relative to ${ }^{42} \mathrm{Ca}$ in the urine at the end of each $24 \mathrm{~h}$ period minus the baseline abundance ratios of ${ }^{44} \mathrm{Ca}$ and ${ }^{43} \mathrm{Ca}$ relative to ${ }^{42} \mathrm{Ca}$. These values were corrected for the natural abundance of each isotope $\left({ }^{44} \mathrm{Ca}=0.02083,{ }^{43} \mathrm{Ca}=0.00135\right)$ and doses (mg) given:

Fractional Ca absorption

$=\left(\left(\left(\frac{{ }^{44} \mathrm{Ca} \Delta \text { excess }}{{ }^{43} \mathrm{Ca} \Delta \text { excess }}\right) \times\left(\frac{0.02083}{0.00135}\right)\right) \times\left(\frac{\text { intravenous dose }}{\text { oral dose }}\right)\right)$.

Balance data were used to calculate Ca retention (equation 2) by subtracting $24 \mathrm{~h}$ Ca excretion in urine and faeces from $24 \mathrm{~h}$ dietary $\mathrm{Ca}$ intake. The first week of each 3-week study was considered to be the equilibration period for the participants to get acclimated to $\mathrm{Ca}$ intake and fibre treatment, while the remaining 2 weeks were considered to be the experimental period. Balance was calculated based on as many of the $14 \mathrm{~d}$ present in the experimental period as possible, so long as it allowed calculations to begin and end on days the faecal samples were collected. The total Ca balance over this period was divided by the number of consecutive days between the faecal sample collection periods to obtain the average daily $\mathrm{Ca}$ balance. Daily urinary Ca excretion values used in the balance calculations were corrected for variation 
in the timing of collections and incomplete collections by adjusting $24 \mathrm{~h}$ urinary $\mathrm{Ca}$ values for daily creatinine excretion (equation 3). Urinary excretion of creatinine remains relatively constant over $24 \mathrm{~h}$, making this metabolite a standard marker of urine sample collection compliance. Ca retention was calculated using both uncorrected and corrected values for urinary $\mathrm{Ca}$ excretion. Apparent $\mathrm{Ca}$ absorption (equation 4) was determined as the difference between $\mathrm{Ca}$ intake and faecal $\mathrm{Ca}$ excretion, while net $\mathrm{Ca}$ absorption efficiency (equation 5) was calculated as $\mathrm{Ca}$ intake minus $\mathrm{Ca}$ faecal excretion, divided by Ca intake:

$$
\begin{aligned}
\mathrm{Ca} \text { retention }= & \text { dietary } \mathrm{Ca} \text { intake }- \text { urinary } \mathrm{Ca} \\
& - \text { faecal } \mathrm{Ca},
\end{aligned}
$$

Corrected $24 \mathrm{~h}$ urinary Ca excretion $=(24 \mathrm{~h}$ urinary $\mathrm{Ca}(\mathrm{mg})) /(24 \mathrm{~h}$ creatinine $(\mathrm{mg}) /$ average $24 \mathrm{~h}$ creatinine excretion for the balance period $(\mathrm{mg}))$,

$$
\begin{aligned}
\text { Apparent } \mathrm{Ca} \text { absorption }= & \text { dietary Ca intake } \\
& - \text { faecal } \mathrm{Ca} \text { excretion, }
\end{aligned}
$$

$$
\begin{aligned}
& \text { Net } \mathrm{Ca} \text { absorption efficiency } \\
& =\frac{\text { dietary } \mathrm{Ca} \text { intake }(\mathrm{mg})-\text { faecal } \mathrm{Ca}(\mathrm{mg})}{\text { dietary Ca intake }} \times 100 \text {. }
\end{aligned}
$$

\section{Faecal sample processing and DNA extraction}

Faecal microbial community composition and structure were determined in samples collected at the beginning and end of each session for each participant. After frozen faecal samples were weighed and completely thawed at $4{ }^{\circ} \mathrm{C}$, sterilised doubledistilled water (twice the weight of the faecal samples) was added to the samples and the samples were homogenised in a stomacher. Faecal slurries were stored at $-20^{\circ} \mathrm{C}$ until DNA extraction. DNA was extracted from $50-100 \mathrm{mg}$ of faecal material using the FastDNA ${ }^{\circledR}$ SPIN Kit for Soil (MP Biochemicals) as described previously ${ }^{(18)}$. DNA quality was checked using a $0.7 \%$ agarose gel and a NanoDrop 1000 spectrophotometer (Thermo Scientific), and then DNA was quantified using a NanoDrop 3300 fluorospectrometer (Thermo Scientific).

\section{Determination of microbial community composition using pyrosequencing}

The phylogenetic diversity of bacterial communities was determined using $16 \mathrm{~S}$ ribosomal RNA (rRNA) gene sequences obtained using 454 FLX titanium chemistry and Roche Genome Sequencer (454 Life Sciences-Roche) and primers that amplify the V3-V5 region of the $16 \mathrm{~S}$ rRNA gene ${ }^{(19)}$. Multiple samples were run and differentiated using $10 \mathrm{bp}$-tagged forward primers. The initial PCR of faecal sample extracts was carried out using high-fidelity Phusion DNA Polymerase (NEB) and amplicons were gel-purified (QIAEX II Gel Extraction Kit, Qiagen). At the Purdue Genomics facilities, purified amplicons were quantified by fluorometry after staining using the PicoGreen DNA Assay Kit (Invitrogen) and by quantitative PCR, and equimolar amounts were used for 454 FLX titanium chemistry sequencing.

The $16 \mathrm{~S}$ rRNA gene sequence dataset was analysed using the Quantitative Insights Into Microbial Ecology (QIIME) pipeline (http://qiime.org/) $)^{(20)}$ that utilises a multi-software approach to perform quality filtering, operational taxonomic unit (OTU) picking, taxonomic assignment, alpha diversity (bacterial diversity within each sample) and beta diversity (bacterial similarities and differences among the samples) measures. With the QIIME pipeline, sequences were split into sample groups using identification tags on the primers and low-quality sequences were removed from the dataset. The quality of the sequences was considered low if the $Q$ score was $<25$ and length was $<400$ or $>1000$ bp or ambiguous sequences or mismatches were found in the primer sequence. OTU were picked using the uclust method $^{(21)}$, and representative OTU sequences were aligned using PyNast (http://biocore.github.io/pynast/) ${ }^{(22)}$ within the QIIME pipeline. ChimeraSlayer ${ }^{(23)}$, a component of the QIIME pipeline, was used to identify and remove chimeric sequences from the OTU table. Final taxonomic assignments for representative OTU were made using the Greengenes dataset version 4feb2011 (http://greengenes.lbl.gov) (97\% sequence similarity) and Ribosomal Database Project (RDP) (http://rdp.cme. msu.edu) classifier at $80 \%$ confidence.

\section{Statistical analyses}

Statistical analyses were carried out using SAS (version 9.2; SAS Institute, Inc.). The baseline characteristics of the female and male participants were compared using $t$ tests. The Wilcoxon rank-sum test was used to assess differences in non-parametric gastrointestinal symptoms. Pearson's correlations were used to examine potential associations between the change in fractional $\mathrm{Ca}$ absorption in the $24-48 \mathrm{~h}$ urine pools (absorption with the SCF treatment minus absorption with the control treatment) and the differences in Ca balance and vitamin D status, baseline anthropometrics, and measures of bone density and strength. A general linear model was used to assess the effect of SCF on fractional Ca absorption. The model controlled for the phase (first $v$. second 3-week camp session) and order of treatments and accounted for the cross-over design by nesting participant identifier (id) within the order of treatments (random-effect variable). The data were analysed separately for each time period (0-24 and 24-48h). Similar analyses were carried out for Ca balance. Using published means and standard deviations for fractional Ca absorption reported in adolescent children, we determined that a sample size of twenty-four children would provide sufficient power (80\%) to observe a 5.9\% difference in fractional Ca absorption assuming an $\alpha$-error of 0.05 and a standard deviation of $9.6 \%{ }^{(24)}$. $P$ values $<0.05$ were considered statistically significant for all statistical tests.

Alpha diversity rarefaction analysis was carried out to estimate total sequence coverage of the community. OTU tables were used to perform alpha biodiversity estimations (e.g. Shannon and Chao1 indices), and within-subject diversity was assessed by pairwise comparisons of the CON 
$v$ SCF samples. Beta biodiversity analysis of the OTU and phylogenetic datasets by 'Fast UniFrac' (phylogenetic distance measure) allowed for the comparison of bacterial community composition between the samples ${ }^{(25)}$. Wilcoxon rank-sum tests were used for pairwise comparisons of the proportions of members of bacterial taxa (all taxonomic levels tested) at the end of the CON $v$. SCF treatments. A $P$ value $<0.05$ was used as the cut-off for statistical significance in bacterial genera analyses. Pearson's correlations and Spearman's rank correlations were used to detect associations between the difference in fractional $\mathrm{Ca}$ absorption between the treatments $(24-48 \mathrm{~h})$ and the difference in the proportions of members of bacterial genera after each treatment. Bacteria used in these correlations were those with a genera average value $>0.001$ ( $=0.1 \%)$, which included the following bacterial taxa: Bifidobacterium; other Coriobacteriaceae; Bacteroides; Barnesiella; Butyricimonas; Parabacteroides; Prevotella; Alistipes; other Rikenellaceae; Enterococcus; Lactobacillus; other Lactobacillaceae; Streptococcus; Clostridium; Eubacterium; Mogibacterium; Blautia; Anaerostipes; Coprococcus; Dorea; other Lachnospiraceae; Roseburia; other Clostridiales; other Peptostreptococcaceae; Sporacetigenium; Acetivibrio; Butyricicoccus; Faecalibacterium; Oscillibacter; other Ruminococcaceae; Ruminococcus; Subdoligranulum; Dialister; other Clostridia; Catenibacterium; Coprobacillus; other Erysipelotrichaceae; Turicibacter; other Firmicutes; other bacteria; Escherichia/ Shigella; Pseudomonas; Actinomyces; other Streptococcaceae; Anaerofustis; Anaerococcus.

\section{Results}

A total of twenty-four adolescents (nine girls and fifteen boys) participated in the present study during the summer of 2010. Among these, three participants did not undergo the fractional $\mathrm{Ca}$ absorption test in both sessions and one participant attended only one of the camp sessions. Therefore, twentyone and twenty-three participants were included in the fractional $\mathrm{Ca}$ absorption and $\mathrm{Ca}$ balance analyses, respectively. Analyses of bone biomarkers and bacterial community proportions included twenty-three participants. The participants evaluated in the present study were ethnically diverse with a distribution of eleven Asian, six Hispanic, one Black and six multi-racial (other) teenagers. The characteristics of the participants including age, anthropometrics, physical parameters and bone measures are reported as means and standard deviations for girls and boys separately in Table 1. Girls and boys had similar physical characteristics; statistically significant differences were observed only in the percentage of lean mass (lower in girls than in boys, $P=0.009$ ) and the percentage of fat mass (higher in girls than in boys, $P=0 \cdot 01$ ) in the study cohort. The mean habitual intakes of $\mathrm{Ca}$ and total fibre and soluble fibre were 768 (SD 403) $\mathrm{mg} / \mathrm{d}$ and 12 (SD 4) g/d and $3.4(\mathrm{SD} 1 \cdot 1) \mathrm{g} / \mathrm{d}$, respectively.

\section{Gastrointestinal symptoms}

No significant differences were observed in gastrointestinal symptom severity between participants on the SCF treatment
Table 1. Baseline characteristics of the participants

(Mean values and standard deviations)

\begin{tabular}{|c|c|c|c|c|}
\hline & \multicolumn{2}{|c|}{ Females ( $n 9)$} & \multicolumn{2}{|c|}{ Males ( $n$ 15) } \\
\hline & Mean & SD & Mean & SD \\
\hline Age (years) & $13 \cdot 3$ & $1 \cdot 0$ & $13 \cdot 5$ & 0.9 \\
\hline Weight (kg) & $59 \cdot 9$ & $13 \cdot 2$ & $61 \cdot 1$ & 11.8 \\
\hline Height $(\mathrm{cm})$ & $157 \cdot 3$ & 4.9 & $164 \cdot 9^{*}$ & $8 \cdot 2$ \\
\hline BMI $\left(\mathrm{kg} / \mathrm{m}^{2}\right)$ & $24 \cdot 1$ & $4 \cdot 0$ & $22 \cdot 4$ & $3 \cdot 1$ \\
\hline BMI percentile (\%) & $80 \cdot 0$ & $16 \cdot 4$ & $74 \cdot 7$ & $19 \cdot 2$ \\
\hline Tanner score average & 3.8 & 0.7 & $2 \cdot 4^{\star \star}$ & 0.9 \\
\hline Lean mass (\%) & $61 \cdot 2$ & $5 \cdot 2$ & $71 \cdot 1^{\star \star}$ & 9.5 \\
\hline Fat mass (\%) & $35 \cdot 1$ & $5 \cdot 6$ & $25 \cdot 1^{*}$ & $10 \cdot 0$ \\
\hline Total body BMD (g/cm²) & 1.07 & 0.11 & 1.04 & 0.11 \\
\hline Total body BMC (g) & 2115 & 329 & 2316 & 424 \\
\hline Total spine BMD $\left(\mathrm{g} / \mathrm{cm}^{2}\right)$ & 1.09 & 0.13 & 1.04 & 0.14 \\
\hline Femoral neck BMD $\left(\mathrm{g} / \mathrm{cm}^{2}\right)$ & 1.03 & 0.18 & 1.05 & 0.15 \\
\hline
\end{tabular}

$\mathrm{BMD}$, bone mineral density; BMC, bone mineral content.

Mean value was significantly different from that of the females: ${ }^{\star} P<0.05$; ${ }^{\star \star} P<0.01$ ( $t$ test).

and those on the CON treatment over the $18 \mathrm{~d}$ observation period. Self-reported mean daily gastrointestinal symptom scores during the SCF and CON treatments were $0 \cdot 1$ (SD $0 \cdot 3$ ) $v .0 .3(\mathrm{SD} 0 \cdot 8)$ for bloating, $0 \cdot 6(\mathrm{SD} 0 \cdot 8) v \cdot 1 \cdot 1$ ( $\mathrm{SD} 1.7)$ for flatulence, $0 \cdot 3(\mathrm{SD} 0.5) v \cdot 0 \cdot 4(\mathrm{SD} 0 \cdot 7)$ for abdominal cramping, and $0.2(\operatorname{sD~} 0.7) v \cdot 0.3(\mathrm{SD} 0.9)$ for stomach noises.

\section{Fractional calcium absorption, calcium balance and bone biomarkers}

Fractional $\mathrm{Ca}$ absorption was based on the analysis of isotopes excreted in the urine, which was collected between 0 and 24 and between 24 and $48 \mathrm{~h}$ after oral and intravenous isotope administration (Fig. 1). Compared with that observed after the CON treatment, mean fractional $\mathrm{Ca}$ absorption did not differ during the first $24 \mathrm{~h}$, but was significantly higher after the SCF treatment $(0.595$ (SD 0.142$) \quad v$. 0.664 (SD 0.129), respectively; $P=0.02$ ) at $24-48 \mathrm{~h}$. The difference in mean fractional Ca absorption of 0.069 occurring $24-48 \mathrm{~h}$ after the treatment represented an $11.6 \%$ increase in absorption with the SCF treatment. A general linear model identified significant effects of treatment on fractional $\mathrm{Ca}$ absorption at $24-48 \mathrm{~h}$ $(P=0.02)$, but not at $0-24 \mathrm{~h}(P=0.09)$. There was no significant effect of treatment order on fractional Ca absorption. Although carry-over effects may have resulted from the 1-week washout period being too short for gut microbiota proportions to return to baseline levels, this finding suggests that the occurrence of carry-over effects was unlikely. There were no significant effects of treatment on net $\mathrm{Ca}$ absorption, net $\mathrm{Ca}$ absorption efficiency, faecal $\mathrm{Ca}$ excretion or Ca retention (Table 2). Values obtained for faecal sample collection compliance, as measured by polyethylene glycol recovery, were 76.0 (SD 12.9) and 78.4 (SD 15.8) \% for the SCF and CON treatments, respectively.

The concentrations of bone turnover markers (Table 3) were measured to explore possible intervention effects on bone turnover. When compared as changes from the baseline, no differences in the concentrations of serum alkaline phosphatase, $\mathrm{P}, \mathrm{Ca}$, parathyroid hormone 1-84, leptin, 


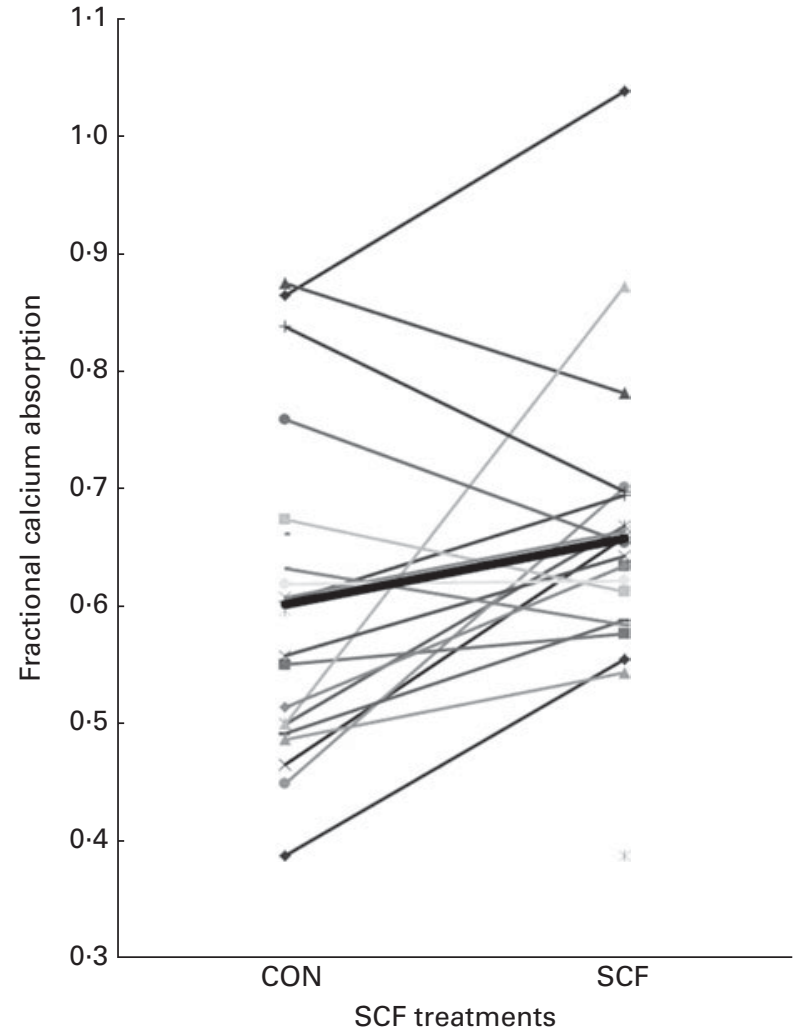

Fig. 1. Comparison of soluble maize fibre (SCF) and control (CON) treatments based on fractional calcium absorption measured with stable isotopes in $24-48 \mathrm{~h}$ urine collections $(n 21)$.

insulin-like growth factor, IGFBP3 and sclerostin and urinary NTX, Ca and P were observed after the SCF treatment.

The mean 25-hydroxyvitamin $\mathrm{D}$ status values after the SCF and CON treatments were 65.2 (SD 18.8) and 59.1 (SD 15.9) nm, respectively, which were not significantly different. No significant associations were observed between differences in vitamin D status and those in fractional Ca absorption (between 24 and $48 \mathrm{~h}$ ) or net Ca absorption efficiency.

The anthropometric parameters and dietary intake variables of the participants that might influence the effect of SCF on lower gut $\mathrm{Ca}$ absorption were evaluated. The difference in fractional $\mathrm{Ca}$ absorption in the $24-48 \mathrm{~h}$ urine collections between participants on the SCF treatment and those on the CON treatment was not correlated with height $(r \quad 0.112$ $P=0.63)$, body surface area $(r$ 0.012, $P=0.96)$, weight $(r-0.022, P=0.92)$, habitual dietary fibre $(r 0.150, P=0.54)$ and $\mathrm{Ca}(r$ 0.012, $P=0.96)$ intakes, Tanner stage $(r-0.131$, $P=0.57)$ or BMI $(r-0.074, P=0.75)$.

\section{Changes in bacterial community composition}

Roche 454-Titanium pyrosequencing of forty-six faecal samples yielded a total of 1793821 16S rRNA sequences with an average of 19498 (SD 7126) (range: 8211-41212) sequences per sample. The number of sequences generated for each participant did not differ significantly when compared by treatment $(P>0.05)$ or the time of faecal collection (baseline $v$. end samples) ( $P>0 \cdot 05)$. Firmicutes was the most dominant phylum with an average of $89.4 \%$ followed by the phyla Bacteroidetes (5.1\%), Actinobacteria (4.9\%) and Proteobacteria (0.5\%) (Fig. 2). Detailed comparisons of microbial community composition in these adolescents have been reported elsewhere $^{(26)}$, and herein we only report differences in community composition after the SCF treatment.

At the phylum level, regardless of the inclusion of SCF in the clinical diet, the average relative proportion of members of the phylum Bacteroidetes significantly increased and that of members of the phylum Firmicutes decreased by the end of each camp session (Fig. 2). The community composition of participants on the SCF treatment was significantly different from that of participants on the CON treatment at the family level (Fig. 3). There were significantly higher proportions of Porphyromonadaceae $(P=0.02)$ and other Clostridiales $(P=0.009)$ and significantly lower proportions of Peptostreptococcaceae $(P=0.04)$ after the SCF treatment. At the end of each 3-week camp session, there were four genera and three 'other' groups that had average proportions that differed significantly $(P<0.05)$ between participants on the SCF treatment and those on the CON treatment (Table 4).

Table 2. Effect of soluble maize fibre (SCF) treatment on calcium absorption and retention in adolescent boys and girls*

(Mean values and standard deviations)

\begin{tabular}{|c|c|c|c|c|c|}
\hline & \multicolumn{2}{|c|}{ SCF } & \multicolumn{2}{|c|}{$\mathrm{CON}$} & \multirow[b]{2}{*}{$P$} \\
\hline & Mean & SD & Mean & SD & \\
\hline \multicolumn{6}{|l|}{ Stable isotope analysis $\dagger$} \\
\hline Fractional Ca absorption $(0-24 \mathrm{~h})$ & 0.522 & 0.110 & 0.497 & $0 \cdot 108$ & 0.09 \\
\hline Fractional $\mathrm{Ca}$ absorption (24-48h) & 0.664 & 0.129 & 0.595 & 0.142 & 0.02 \\
\hline \multicolumn{6}{|l|}{ Balance analysis $\ddagger$} \\
\hline Ca intake $(\mathrm{mg} / \mathrm{d})$ & 606 & 29 & 604 & 25 & 0.69 \\
\hline Urinary Ca (mg/d) & 77 & 56 & 65 & 36 & 0.11 \\
\hline Faecal Ca (mg/d) & 318 & 108 & 312 & 106 & 0.77 \\
\hline Net Ca absorption efficiency (\%) & 47 & 18 & 48 & 17 & 0.75 \\
\hline Ca retention (mg/d) & 212 & 117 & 227 & 101 & 0.42 \\
\hline
\end{tabular}

CON, control.

* General linear model that includes treatment, order and phase.

†For fractional $\mathrm{Ca}$ absorption calculations, $n 21$ (three subjects did not undergo both fractional $\mathrm{Ca}$ absorption tests and were, therefore, excluded from the analysis).

‡For balance calculations, $n 23$ (one subject completed only one treatment phase). 
Table 3. Serum and urinary bone turnover marker concentrations at baseline and after treatment with 0 and $12 \mathrm{~g}$ soluble maize fibre (SCF)*

(Mean values and standard deviations, $n$ 23)

\begin{tabular}{|c|c|c|c|c|c|c|c|}
\hline \multirow[b]{2}{*}{ Bone biomarkers } & \multicolumn{2}{|c|}{ Baseline } & \multicolumn{2}{|c|}{ SCF } & \multicolumn{2}{|c|}{ CON } & \multirow[b]{2}{*}{$P \dagger$} \\
\hline & Mean & SD & Mean & SD & Mean & SD & \\
\hline \multicolumn{8}{|l|}{ Serum } \\
\hline Alkaline phosphatase $(\mathrm{U} / \mathrm{l})$ & $237 \cdot 29$ & $125 \cdot 80$ & 235.57 & 135.45 & 235.00 & $132 \cdot 5$ & 0.50 \\
\hline $\mathrm{Ca}(\mathrm{ng} / \mathrm{l})$ & $105 \cdot 1$ & $4 \cdot 1$ & $102 \cdot 3$ & 4.0 & $102 \cdot 4$ & $4 \cdot 0$ & 0.54 \\
\hline Creatinine (ng/l) & 8.5 & $1 \cdot 1$ & 8.5 & $1 \cdot 2$ & 8.5 & $1 \cdot 2$ & 0.66 \\
\hline $\mathrm{P}(\mathrm{ng} / \mathrm{l})$ & $48 \cdot 6$ & $5 \cdot 4$ & $47 \cdot 6$ & 5.9 & $47 \cdot 6$ & $5 \cdot 8$ & 0.21 \\
\hline PTH (pg/ml) & $18 \cdot 20$ & $7 \cdot 34$ & $21 \cdot 23$ & $11 \cdot 27$ & 21.23 & 11.02 & 0.50 \\
\hline Leptin (ng/ml) & $10 \cdot 17$ & $7 \cdot 77$ & 8.65 & $7 \cdot 61$ & 8.46 & $7 \cdot 50$ & 0.83 \\
\hline IGF-1 (ng/ml) & 296.53 & 53.97 & $266 \cdot 81$ & $52 \cdot 30$ & 269.59 & 52.93 & 0.87 \\
\hline IGFBP3 (ng/ml) & 3879.88 & 554.63 & 3648.99 & $572 \cdot 71$ & $3651 \cdot 26$ & $560 \cdot 23$ & 0.46 \\
\hline Sclerostin (ng/ml) & 0.45 & 0.23 & 0.41 & 0.17 & 0.41 & 0.17 & 0.48 \\
\hline \multicolumn{8}{|l|}{ Urine } \\
\hline NTX (nmol BCE/l) & 2973.47 & 1788.00 & $5378 \cdot 81$ & 4632.47 & 5323.75 & 4538.67 & 0.76 \\
\hline $\mathrm{Ca}(\mathrm{mg} / \mathrm{l})$ & 39.1 & $27 \cdot 9$ & 34.3 & $25 \cdot 1$ & $33 \cdot 7$ & 24.7 & 0.71 \\
\hline $\mathrm{P}(\mathrm{mg} / \mathrm{l})$ & $522 \cdot 7$ & $205 \cdot 1$ & 778.5 & $399 \cdot 7$ & $766 \cdot 9$ & $395 \cdot 1$ & 0.88 \\
\hline Creatinine $(\mathrm{mg} / \mathrm{l})$ & 586.9 & 218.5 & $903 \cdot 0$ & $457 \cdot 2$ & $906 \cdot 7$ & 447.5 & 0.38 \\
\hline
\end{tabular}

Significant increases in proportions after the SCF treatment were observed for the genera Parabacteroides $(P<0.003)$, other Clostridiales $(P=0.04)$ and other Ruminococcaceae $(P<0.03)$, while significant decreases in proportions were observed for Enterococcus $(P<0.03)$, Anaerofustis $(P<0.05)$, Coprococcus $(P<0.03)$ and other Peptostreptococcaceae $(P<0 \cdot 002)$. There was also an increase in the proportions of Bifidobacterium, Alistipes, Anaerococcus, Catenibacterium and other Clostridia, but the increase in the proportions was not significant. Similarly, decreases in the proportions of Rothia, other Streptococcaceae, Clostridium, Sporacetigenium, Turicibacter and other TM7 genera Incertae sedis were observed after the SCF treatment, but the difference in community proportion after the CON treatment was not significant. Fast UniFrac with jackknife analysis revealed no differences in community structure due to the overall treatment, despite the data being processed using different classification criteria (e.g. OTU $v$. phylogeny) (data not shown). Alpha diversity values also did not differ significantly between participants on the SCF treatment and those on the CON treatment (data not shown)

\section{Correlations between the proportions of members of bacterial genera and fractional calcium absorption}

In an exploratory analysis, Pearson's correlations and Spearman rank-sum analyses were used to identify bacteria having potential influence on the $\mathrm{Ca}$ absorption response. Changes (those with the SCF treatment minus those with the CON treatment) in fractional $\mathrm{Ca}$ absorption measured in the $24-48 \mathrm{~h}$ urine collections were negatively correlated (decreases in the proportions of members of bacterial genera as $\mathrm{Ca}$ absorption with the SCF treatment increased) with the proportions of Actinomyces, Pseudomonas of the phylum Actinobacteria and other Erysipelotrichaceae of the phylum Firmicutes. Conversely, changes in fractional Ca absorption were positively correlated (increases in the proportions of members of bacterial genera as $\mathrm{Ca}$ absorption with the SCF treatment increased) with the proportions of the Bacteroidetes member Bacteroides as well as Butyricicoccus, Oscillibacter and Dialister of the phylum Firmicutes (Table 5).

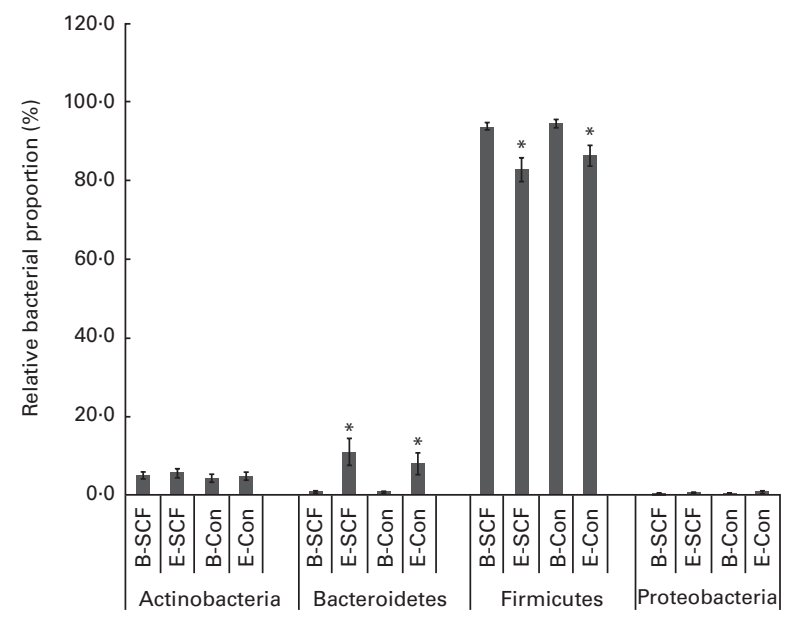

Fig. 2. Comparison of the average relative proportions of members of bacterial phyla in participants at the beginning $(B)$ and end $(E)$ of the clinical sessions where diets included soluble maize fibre (SCF) $v$. control (CON). Only phyla representing $>0.5 \%$ of the total community during at least one treatment are depicted. Values are means, with their standard errors represented by vertical bars. * Mean value was significantly different from that of the B sample within each phylum $(P<0.05)$. Significant differences in bacterial phyla between participants on the SCF treatment and those on the CON treatment at $E$ were not apparent. 


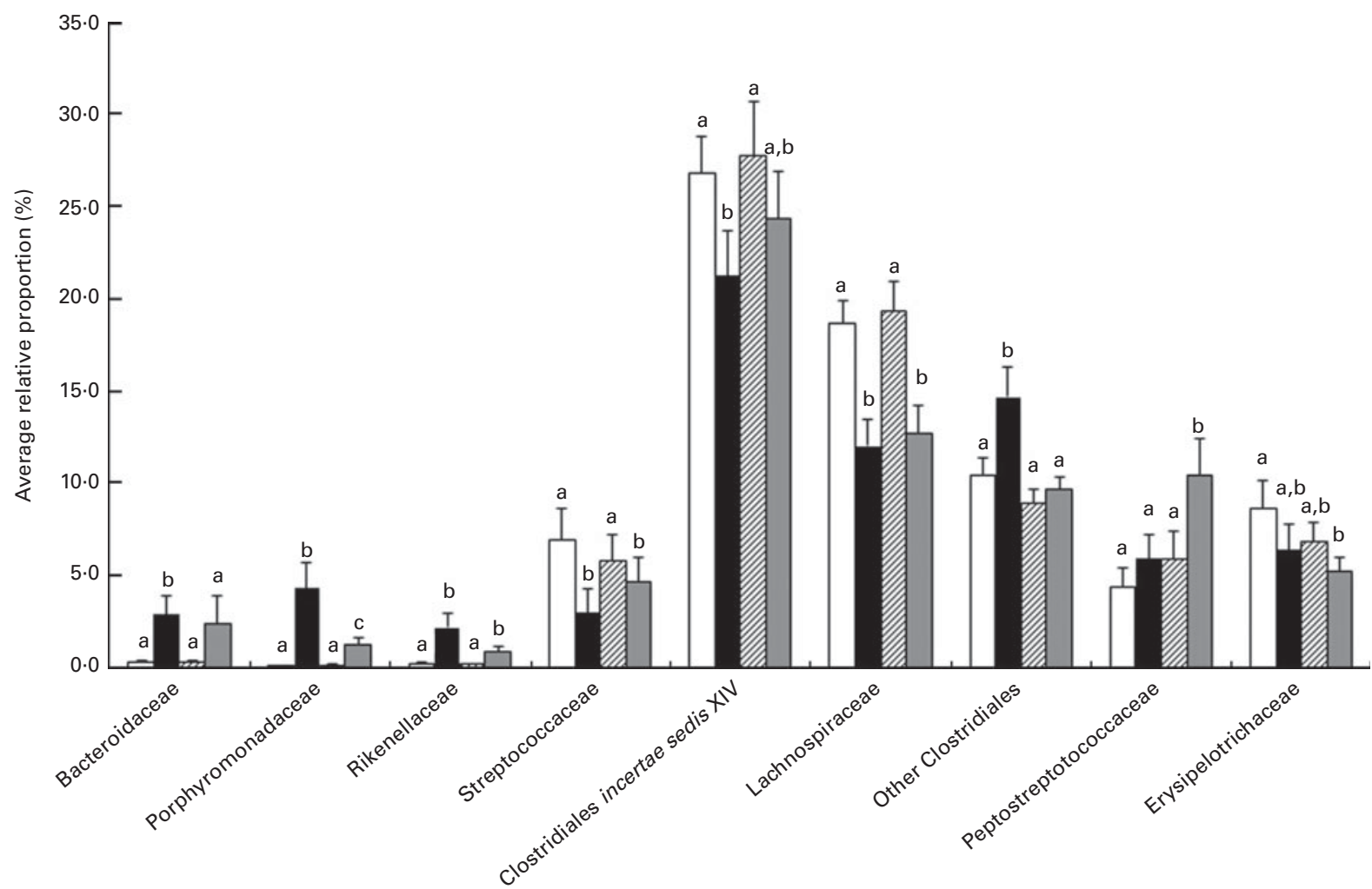

Fig. 3. Comparison of the average relative proportions of members of bacterial families in participants ( $n$ 23) at the beginning (B) and end (E) of the clinical sessions where diets included soluble maize fibre (SCF) v. control (CON). At time E, significant differences in the proportions of Bacteroidaceae, Porphyromonadaceae, other Clostridiales and Peptostreptococcaceae were observed between participants on the SCF treatment and those on the CON treatment. Only families representing $>1.0 \%$ of the total community during at least one treatment and exhibiting significant differences in relative proportions are shown. Values are means, with their standard errors represented by vertical bars. ${ }^{a, b, c}$ Mean values with unlike letters were significantly different within each family $(P<0 \cdot 05)$. $\square$, SCF-B; $\boldsymbol{\square}$, SCF-E; $\square$, CON-B; $\square$, CON-E.

\section{Discussion}

Significant increases in fractional $\mathrm{Ca}$ absorption were observed in adolescent boys and girls consuming SCF. Broadly, the consumption of SCF resulted in lower proportions of faecal bacteria of the phylum Firmicutes and greater proportions of bacteria of the phylum Bacteroidetes. These changes may influence bone health as increases in fractional $\mathrm{Ca}$ absorption were negatively correlated with the proportions of members of the genera Actinomyces and Pseudomonas and positively correlated with those of the genera Bacteroides, Butyricicoccus, Oscillibacter and Dialister. Despite the evidence for an acute increase in fractional $\mathrm{Ca}$ absorption via faecal microbiota, no effect of SCF on Ca balance or biochemical markers of bone turnover was observed.

In the present study, daily consumption of $12 \mathrm{~g}$ SCF for $21 \mathrm{~d}$ by adolescent girls and boys increased fractional $\mathrm{Ca}$ absorption by approximately $12 \%$. This effect was similar to that observed in a previous study reporting a $10.8 \%$ difference in true Ca absorption in adolescent boys consuming $15 \mathrm{~g} / \mathrm{d}$ oligofructose for $9 \mathrm{~d}$ compared with the control group ${ }^{(17)}$. Using dual-stable isotope methods, increases in fractional Ca absorption relative to those observed after control treatments were found to range from 3 to $18 \%$ in young girls and boys consuming $8 \mathrm{~g} / \mathrm{d}$ inulin-oligofructose mix (Synergy $\left.1^{(\circledR)}\right)^{(3,4,24,27)}$. In the present study, habitual fibre intake, specifically of soluble fibre, was low. This may have influenced the response to SCF intervention observed in these teenagers, as positive effects of inulin-type fructans on $\mathrm{Ca}$ absorption were only observed when the habitual basal diet lacked fermentable fibres ${ }^{(28)}$.

The $12 \%$ increase in fractional $\mathrm{Ca}$ absorption observed in the present study occurred between 24 and $48 \mathrm{~h}$ as the effect was significant when measuring the second $24 \mathrm{~h}$ urine pool $(24-48 \mathrm{~h})$ after the administration of stable Ca isotopes. No significant difference was observed in isotope enrichment in the urine samples collected during the first $24 \mathrm{~h}$. This finding is supported by the literature that suggests that microbial involvement and lower gut absorption are not realised until $24 \mathrm{~h}$ after isotope administration ${ }^{(17)}$. Other studies have not measured fractional $\mathrm{Ca}$ absorption at various time intervals, as has been done in the present study, to evaluate the effects of prebiotics on $\mathrm{Ca}$ absorption over time.

Urinary Ca excretion was non-significantly $18 \%$ higher after the SCF treatment compared with that after the CON treatment, reflective of a greater fractional $\mathrm{Ca}$ absorption efficiency. An increase in urinary Ca excretion could contribute to the lack of a beneficial effect of SCF on net Ca absorption and net $\mathrm{Ca}$ balance. However, because the increase in urinary Ca excretion was not significant, it is more likely that differences in methods explain the inconsistencies observed in the benefits conferred by SCF. Fractional Ca absorption reveals 
Table 4. Comparison of the average proportions (\%) of members of bacterial taxa in the faecal samples of participants at the end of the soluble maize fibre (SCF) treatment $v$. control (CON) treatment

(Mean values with their standard errors, $n 23$ )

\begin{tabular}{|c|c|c|c|c|c|}
\hline \multirow[b]{2}{*}{ Taxon } & \multicolumn{2}{|c|}{ SCF } & \multicolumn{2}{|c|}{ CON } & \multirow[b]{2}{*}{$P^{*}$} \\
\hline & Mean & SEM & Mean & SEM & \\
\hline \multicolumn{6}{|l|}{ Phylum: Bacteroidetes } \\
\hline \multicolumn{6}{|c|}{ Class: Bacteroidia; Order: Bacteroidales; Family: Porphyromonadaceae } \\
\hline Genus: Parabacteroides & 3.58 & $1 \cdot 18$ & 0.83 & 0.32 & 0.003 \\
\hline \multicolumn{6}{|l|}{ Phylum: Firmicutes } \\
\hline \multicolumn{6}{|c|}{ Class: Bacilli; Order: Lactobacillales; Family: Enterococcaceae } \\
\hline Genus: Enterococcus & 0.012 & 0.037 & 0.590 & $2 \cdot 100$ & 0.027 \\
\hline \multicolumn{6}{|c|}{ Class: Clostridia; Order: Clostridiales; Family: Eubacteriaceae } \\
\hline Genus: Anaerofustis & 0.006 & 0.002 & 0.012 & 0.004 & 0.048 \\
\hline \multicolumn{6}{|c|}{ Class: Clostridia; Order: Clostridiales; Family: Lachnospiraceae } \\
\hline Genus: Coprococcus & 0.68 & 0.13 & $1 \cdot 15$ & 0.25 & 0.027 \\
\hline \multicolumn{6}{|l|}{ Class: Clostridia } \\
\hline Order: other Clostridiales & $14 \cdot 64$ & 1.63 & $9 \cdot 61$ & 0.69 & 0.013 \\
\hline \multicolumn{6}{|l|}{ Class: Clostridia; Order: Clostridiales; } \\
\hline Family: other Peptostreptococcaceae & 0.42 & 0.10 & 1.00 & 0.18 & 0.001 \\
\hline \multicolumn{6}{|l|}{ Class: Clostridia; Order: Clostridiales } \\
\hline Family: other Ruminococcaceae & 4.07 & 1.02 & 1.93 & 0.45 & 0.030 \\
\hline
\end{tabular}

* Only taxa in which proportions at the end of the treatment differed with $P<0.05$ (Wilcoxon's rank-sum test) are listed.

small changes in isotopic tracer levels over a short period of time and $\mathrm{Ca}$ balance is a more crude measure of total Ca content over a longer period of time. We powered the study for fractional $\mathrm{Ca}$ absorption, a more sensitive measure than $\mathrm{Ca}$ retention, with respect to measurement variability compared with $\mathrm{Ca}$ retention and net $\mathrm{Ca}$ absorption. For example, faecal $\mathrm{Ca}$ excretion is highly variable, requiring a sample size of thirty-four subjects to detect a $61 \mathrm{mg}$ difference in $\mathrm{Ca}$ retention with an $\alpha$-error of $0 \cdot 05,80 \%$ power and a standard deviation of the difference of $122 \mathrm{mg} / \mathrm{d}$ between the participants. Despite the lack of power to detect an effect on $\mathrm{Ca}$ retention, it is possible that the SCF treatment exerted an effect on bone strength. Weaver et al. ${ }^{(12)}$ reported an increase in the peak breaking force of the femur, but no change in retention in rats consuming $4 \%$ SCF by weight. These two methods have a large difference in the percentage of standard deviation, i.e. $9.7 \%$ for breaking force and $41.3 \%$ for Ca retention. This change in bone strength suggests that the SCF intervention perturbed Ca balance in order to affect bone balance.

Consistent with a beneficial effect of fibre consumption on bone, in a long-term study carried out in adolescent boys and girls, consumption of $8 \mathrm{~g} / \mathrm{d}$ of an inulin-type fructan mixture was found to result in a significantly increased BMD and a persistent effect on fractional $\mathrm{Ca}$ absorption from 8 weeks ( $8.5 \%)$ to 1 year $(5.9 \%)$ of daily consumption with no difference being observed in urinary $\mathrm{Ca}$ excretion ${ }^{(3)}$. An animal study carried out in our laboratory specifically demonstrated an increased trabecular BMD in addition to increased cortical area and thickness in the femur after the consumption of SCF ( $4 \%$ by weight of the diet) ${ }^{(12)}$. We observed no differences in the biochemical markers of bone turnover. In small metabolic studies, the high variance in bone markers usually does not result in significant changes in bone turnover due to nutritional interventions that are apparent with isotopic tracer kinetic approaches ${ }^{(29)}$. Thus, it is more likely that the variability in Ca balance measures observed in the present study was too high to detect effects of higher Ca absorption. Longer-term studies of bone strength and density are needed to determine the beneficial effects of SCF consumption on bone, as physiological effects (Ca retention and bone turnover) have not been detected. Assuming that the increased absorption of $\mathrm{Ca}$ with the SCF treatment is sustained, data obtained in the present study indicate that treatment with SCF would lead to an additional Ca retention of $41.4 \mathrm{mg} / \mathrm{d}$. Persistence over a year would account for an additional $15.1 \mathrm{~g}$ of $\mathrm{Ca}$ or $1.8 \%$ of total body $\mathrm{Ca}$ assuming the mean bone mineral content, measured by dual-energy X-ray absorptiometry, in the study cohort (2241 g).

The increases in the proportions of members of the phylum Bacteroidetes (Parabacteroides and Alistipes) and a trend

Table 5. Correlations* between calcium absorption and proportions of members of bacterial genera that may affect lower gut mechanisms (n 21)†

\begin{tabular}{lcc}
\hline & \multicolumn{2}{c}{$\begin{array}{c}\text { Difference in Ca } \\
\text { absorption at 24-48 h }\end{array}$} \\
\cline { 2 - 3 } $\begin{array}{l}\text { Differences in the proportions of members } \\
\text { of genera at the end of each treatment }\end{array}$ & Coefficient & $P$ \\
\hline Bacteroides & 0.483 & 0.027 \\
Actinomyces & -0.553 & 0.009 \\
Pseudomonas & -0.473 & 0.03 \\
Butyricicoccus & 0.454 & 0.039 \\
Oscillibacter & 0.565 & 0.008 \\
Dialister & 0.619 & 0.003 \\
Other Erysipelotrichaceae & -0.463 & 0.034 \\
\hline
\end{tabular}

*Pearson's correlations and Spearman's rank correlations.

†Data are shown for correlations that were significant $(P<0.05)$, while analysis included correlations for all genera making up $>0.001(=0.1 \%)$, which included the following bacterial taxa: Bifidobacterium; other Coriobacteriaceae; Bacteroides; Barnesiella; Butyricimonas; Parabacteroides; Prevotella; Alistipes; other Rikenellaceae; Enterococcus; Lactobacillus; other Lactobacillaceae; Streptococcus; Clostridium; Eubacterium; Mogibacterium; Blautia; Anaerostipes; Coprococcus; Dorea; other Lachnospiraceae; Roseburia; other Clostridiales; other Peptostreptococcaceae; Sporacetigenium; Acetivibrio; Butyricicoccus; Faecalibacterium; Oscillibacter, other Ruminococcaceae; Ruminococcus; Subdoligranulum; Dialister, other Clostridia; Catenibacterium; Coprobacillus; other Erysipelotrichaceae; Turicibacter, other Firmicutes; other bacteria; Escherichia/ Shigella; Pseudomonas; Actinomyces; other Streptococcaceae; Anaerofustis; Anaerococcus. 
towards a greater increase in the proportion of Bifidobacterium suggest that the inclusion of SCF in the diet increased the proportion of beneficial bacteria capable of fermenting RS and producing $\mathrm{SCFA}^{(30)}$. The interesting finding that the proportions of members of the phylum Bacteroidetes increased significantly on both the SCF and CON treatments was more likely due to a response to the control diet rather than to a carry-over effect of the SCF treatment. This is supported by the lack of a significant order effect on study outcomes and is further supported by the control diet providing more dietary fibre than the participants' habitual diets. Significant increases in the proportions of members of the family Bacteroidaceae have been reported in adult men after the consumption of SCF as a part of their regular diet ${ }^{(31)}$, but curiously in this particular study the proportion of Bifidobacterium significantly decreased compared with that in the control group. Conversely, healthy adults consuming four increasing doses of galacto-oligosaccharides $(0-10 \mathrm{~g})$ were found to exhibit dosedependent increases in the proportion of bifidobacteria after 3 weeks of consumption ${ }^{(32)}$. In addition to the previously mentioned genera, changes in the proportions of Butyricicoccus, Oscillibacter and Dialister were also significantly correlated with increased $\mathrm{Ca}$ absorption in the present study. These genera have previously been identified for their production of SCFA, which may be the result of fibre fermentation ${ }^{(33,34,35)}$.

Conversely, the significant decreases in the proportions of members of the genera Enterococcus, Anaerofustis, Coprococcus and other Peptostreptococcaceae with the SCF treatment are less understood, as their functional roles in Ca metabolism have not been identified yet. After fibre is fermented to acetate or lactate, a number of bacteria, which are often members of the phylum Firmicutes, can continue fermenting these metabolites to butyrate. This process may be carried out by other Clostridia and other Ruminococcaceae, taxa that are currently unclassified, the proportions of which increased significantly with the SCF treatment. However, there is very little functional information available currently on these members of the phylum Firmicutes to support this hypothesis. Another study comparing the effect of two different types of RS has reported some similar findings; the authors demonstrated an increase in the proportions of Parabacteroides, Bacteroides, Clostridium clostridioforme and other Clostridiales in participants given a RS4 diet and an increase in those of Eubacterium and Ruminococcus in participants given a RS2-enhanced diet ${ }^{(36)}$. They also found decreases in the proportions of known butyrate producers Faecalibacterium and Dorea. Inclusion of RS3 in the diet of overweight males was found to result in an increase in the proportions of Oscillibacter, Eubacterium and Ruminococcus $^{(37)}$. Similar to the present study, these studies could identify specific taxa associated with the dietary treatment, but had difficulty in distinguishing the effect of diet on overall community structure (e.g. using UniFrac) probably due to inter-subject variability in species that are affected and their ability to utilise substrates ${ }^{(36,37)}$.

Some limitations of the present study are the use of only one level of SCF treatment and one level of Ca intake, because work in rats has suggested that the effects of oligofructose on bone structure vary depending on $\mathrm{Ca}$ intake ${ }^{(9)}$. Thus, neither a dose-response effect of treatment nor the influence of $\mathrm{Ca}$ intake on response could be determined. The short duration of the study was also one of the limitations, as we were unable to assess changes in bone density and strength, which a longer-duration study, such as the 1-year intervention study carried out by Abrams et al. ${ }^{(3)}$, could have assessed. A future randomised controlled trial is warranted to determine the effects of long-term consumption of SCF on BMD, bone mineral content and bone geometry and associated changes in microbiota.

In conclusion, consumption of $12 \mathrm{~g} / \mathrm{d} \mathrm{SCF}$, a prebiotic fibre that was well tolerated in the present study, positively influenced short-term Ca absorption in adolescent girls and boys. SCF-induced absorption occurred after $24 \mathrm{~h}$, which may be indicative of lower gut involvement as this change coincided with increases in the proportions of SCFA-producing bacteria. However, SCF did not alter net Ca retention. Therefore, the present study cannot resolve whether the balance method is too insensitive to determine the benefits of improved Ca absorption or whether the benefits decline with chronic feeding. Further work is required to assess whether SCF influences bone mineralisation in the long term and how microbial interplay modulates the individual response to prebiotic treatments.

\section{Acknowledgements}

The authors thank Arthur Armstrong and Andrea Clavijo for performing the PCR-denaturing gradient gel electrophoresis and pyrosequencing analyses and for their assistance in interpreting sequence data. This project would not have been possible without the longstanding technical and clinical assistance of Ania Kempa-Steczko and Doug Maish.

The present study was funded by Tate \& Lyle. In addition to financial support, Tate \& Lyle contributed to the study design and reviewed the manuscript. The data obtained in the course of the present study and reported in the article are considered proprietary data to which Tate \& Lyle claims 'exclusive right of reference' in accordance with Regulation (EC) no. 1924/2006 of the European Parliament and of the Council on Nutrition and Health Claims Made on Foods. The 'exclusive right of reference' does not prohibit the referencing or quotation of the present article, provided any such reference or quotation does not call into question Tate \& Lyle's ownership of data. The content of the present article does not constitute an endorsement of any product of Tate \& Lyle by Purdue.

C. M. Weaver serves on the Advisory Board of Pharmavite.

The authors' contributions are as follows: C. M. Weaver, C. H. N. and B. R. M. designed the research; B. R. M., C. H. N. and C. M. Whisner conducted the research; C. M. Whisner, C. H. N., L. D. M. and G. P. M. analysed the data; C. M. Whisner, C. H. N. and C. M. Weaver wrote the paper; C. M. Weaver had primary responsibility for the final content. All authors read and approved the final manuscript.

\section{References}

1. Fulgoni VL III, Keast DR, Auestad N, et al. (2011) Nutrients from dairy foods are difficult to replace in diets of Americans: food pattern modeling and an analyses of the National 
Health and Nutrition Examination Survey 2003-2006. Nutr Res 31, 759-765.

2. Krebs-Smith SM, Guenther PM, Subar AF, et al. (2010) Americans do not meet federal dietary recommendations. $J$ Nutr 140, 1832-1838.

3. Abrams SA, Griffin IJ, Hawthorne KM, et al. (2005) A combination of prebiotic short- and long-chain inulin-type fructans enhances calcium absorption and bone mineralization in young adolescents. Am J Clin Nutr 82, 471-476.

4. Griffin IJ, Davila PM \& Abrams SA (2002) Non-digestible oligosaccharides and calcium absorption in girls with adequate calcium intakes. Br J Nutr 87, Suppl. 2, S187-S191.

5. van den Heuvel EG, Schoterman MH \& Muijs T (2000) Transgalactooligosaccharides stimulate calcium absorption in postmenopausal women. J Nutr 130, 2938-2942.

6. Devareddy L, Khalil DA, Korlagunta K, et al. (2006) The effects of fructo-oligosaccharides in combination with soy protein on bone in osteopenic ovariectomized rats. Menopause 13, 692-699.

7. Weaver CM, Martin BR, Nakatsu CH, et al. (2011) Galactooligosaccharides improve mineral absorption and bone properties in growing rats through gut fermentation. J Agric Food Chem 59, 6501-6510.

8. Zafar TA, Weaver CM, Zhao Y, et al. (2004) Nondigestible oligosaccharides increase calcium absorption and suppress bone resorption in ovariectomized rats. J Nutr 134, 399-402.

9. Scholz-Ahrens KE, Acil Y \& Schrezenmeir J (2002) Effect of oligofructose or dietary calcium on repeated calcium and phosphorus balances, bone mineralization and trabecular structure in ovariectomized rats. Br J Nutr 88, 365-377.

10. Lobo AR, Colli C \& Filisetti TMCC (2006) Fructooligosaccharides improve bone mass and biomechanical properties in rats. Nutr Res 26, 413-420.

11. Bongers A \& Van den Heuvel EG (2003) Prebiotics and the bioavailability of minerals and trace elements. Food Rev Int 19, 397-422.

12. Weaver CM, Martin BR, Story JA, et al. (2010) Novel fibers increase bone calcium content and strength beyond efficiency of large intestine fermentation. J Agric Food Chem 58, 8952-8957.

13. Harris J \& Benedict F (1919) A Biometric Study of Basal Metabolism in Man. Washington: Carnegie Institute of Washington.

14. Wu L, Martin BR, Braun MM, et al. (2010) Calcium requirements and metabolism in Chinese-American boys and girls. J Bone Miner Res 25, 1842-1849.

15. Allen LH, Raynolds WL \& Margen S (1979) Polyethylene glycol as a quantitative fecal marker in human nutrition experiments. Am J Clin Nutr 32, 427-440.

16. Whisner CM, Martin BR, Schoterman MH, et al. (2013) Galacto-oligosaccharides increase calcium absorption and gut bifidobacteria in young girls: a double-blind cross-over trial. Br J Nutr 110, 1292-1303.

17. van den Heuvel EG, Muys T, van Dokkum W, et al. (1999) Oligofructose stimulates calcium absorption in adolescents. Am J Clin Nutr 69, 544-548.

18. Ariefdjohan MW, Savaiano DA \& Nakatsu CH (2010) Optimization of DNA extraction kits for PCR-DGGE analysis of human intestinal microbial communities from fecal specimens. Nutr J 9, 23.

19. Nossa CW, Oberdorf WE, Yang L, et al. (2010) Design of $16 \mathrm{~S}$ rRNA gene primers for 454 pyrosequencing of the human foregut microbiome. World $J$ Gastroenterol 16, 4135-4144.
20. Caporaso JG, Kuczynski J, Stombaugh J, et al. (2010) QIIME allows analysis of high-throughput community sequencing data. Nat Meth 7, 335-336.

21. Edgar RC (2010) Search and clustering orders of magnitude faster than BLAST. Bioinformatics 26, 2460-2461.

22. Caporaso JG, Bittinger K, Bushman FD, et al. (2010) PyNAST: a flexible tool for aligning sequences to a template alignment. Bioinformatics 26, 266-267.

23. Haas BJ, Gevers D, Earl AM, et al. (2011) Chimeric 16S rRNA sequence formation and detection in Sanger and 454-pyrosequenced PCR amplicons. Genome Res 21, 494-504.

24. Griffin IJ, Hicks PDM, Heaney RP, et al. (2003) Enriched chicory inulin increases Ca absorption mainly in girls with lower Ca absorption. Nutr Res 23, 901-909.

25. Hamady M, Lozupone C \& Knight R (2010) Fast UniFrac: facilitating high-throughput phylogenetic analyses of microbial communities including analysis of pyrosequencing and PhyloChip data. ISME J 4, 17-27.

26. Clavijo-Gutierrez AP (2013) Response of human gut microbiota to diet supplementation with soy or soluble corn fiber. PhD Thesis, Purdue University

27. Abrams SA, Griffin IJ \& Hawthorne KM (2007) Young adolescents who respond to an inulin-type fructan substantially increase total absorbed calcium and daily calcium accretion to the skeleton. J Nutr 137, 2524S-2526S.

28. Raschka L \& Daniel H (2005) Diet composition and age determine the effects of inulin-type fructans on intestinal calcium absorption in rat. Eur J Nutr 44, 360-364.

29. Wastney ME, Martin BR, Peacock M, et al. (2000) Changes in calcium kinetics in adolescent girls induced by high calcium intake. J Clin Endocrinol Metab 85, 4470-4475.

30. Maathuis A, Hoffman A, Evans A, et al. (2009) The effect of the undigested fraction of maize products on the activity and composition of the microbiota determined in a dynamic in vitro model of the human proximal large intestine. J Am Coll Nutr 28, 657-666.

31. Hooda S, Boler BM, Serao MC, et al. (2012) 454 pyrosequencing reveals a shift in fecal microbiota of healthy adult men consuming polydextrose or soluble corn fiber. J Nutr $\mathbf{1 4 2}$, $1259-1265$.

32. Davis LM, Martinez I, Walter J, et al. (2010) A dose dependent impact of prebiotic galactooligosaccharides on the intestinal microbiota of healthy adults. Int J Food Microbiol 144, 285-292.

33. Eeckhaut V, Van Immerseel F, Teirlynck E, et al. (2008) Butyricicoccus pullicaecorum gen. nov., sp. nov., an anaerobic, butyrate-producing bacterium isolated from the caecal content of a broiler chicken. Int J Syst Evol Microbiol 58, $2799-2802$.

34. Iino T, Mori K, Tanaka K, et al. (2007) Oscillibacter valericigenes gen. nov., sp. nov., a valerate-producing anaerobic bacterium isolated from the alimentary canal of a Japanese corbicula clam. Int J Syst Evol Microbiol 57, $1840-1845$.

35. Jumas-Bilak E, Jean-Pierre H, Carlier JP, et al. (2005) Dialister micraerophilus sp. nov. and Dialister propionicifaciens sp. nov, isolated from human clinical samples. Int J Syst Evol Microbiol 55, 2471-2478.

36. Martinez I, Kim J, Duffy PR, et al. (2010) Resistant starches types 2 and 4 have differential effects on the composition of the fecal microbiota in human subjects. PLOS ONE $\mathbf{5}$, e15046.

37. Walker AW, Ince J, Duncan SH, et al. (2011) Dominant and diet-responsive groups of bacteria within the human colonic microbiota. ISME J 5, 220-230. 